\title{
Reducing Energy Consumption in an Industrial Process by Using Model Predictive Control
}

\author{
Luis Caiza*, Albert Rosich-Oliva, Carlos Ocampo-Martínez ${ }^{\dagger}$ and Diego S. Benítez* \\ *Colegio de Ciencias e Ingenierías "El Politécnico", \\ Universidad San Francisco de Quito USFQ, Campus Cumbayá, Casilla Postal 17-1200-841, Quito, Ecuador
}

†Institut de Robótica i Informática Industrial, Departamento de Ingeniería de Sistemas, Automática e Informática Industrial, Universitat Politécnica de Catalunya, Parque Tecnológico de Barcelona. C/ Llorens y Artigas 4-6. 08028, Barcelona, España email: \{lcaiza, dbenitez\}@usfq.edu.ec, \{arosich, cocampo\}@iri.upc.edu

\begin{abstract}
In this paper, we propose to use Model Predictive Control techniques to reduce the energy consumption in an industrial process by incorporating energy consumption restrictions in the problem formulation. We propose to use a Model Predictive Control supervisor to calculate the optimum references for simple control loops regulated by individual PID controllers. The results obtained are compared against those obtained when a traditional control strategy exclusively based on PID controllers was used. By using this supervisory control strategy a reduction on energy consumption of about $10 \%$ was achieved.
\end{abstract}

Index Terms-Model Predictive Control, Energy Reduction, System Identification, PID control.

\section{INTRODUCTION}

In the past, the control of industrial processes was mainly based on maintaining a stable operation and reducing the influence of external disturbances. To achieve these objectives, proportional integral derivative (PID) controllers have been successfully used. However, nowadays due to the globalization of markets, the changing needs and demands from customers, and the growing social interest in environmental problems related to production processes, industries are also forced to adopt control strategies to remain competitive and profitable. One powerful tools to address these challenges is Model Predictive Control (MPC) [1]. MPC control allows to also incorporate economic and environmental criteria in the control strategy, since the modeling of the problem may accept any type of models, objective functions and restrictions, thus improving therefore the production results from the economic point of view and quality in the industry process. On the other hand, industrial processes are always complex. For example, the presence of delay times and physical constraints specific to the nature of the system, and the behavior of its variables, need to be considered by the control strategy. The design of a predictive controller allows to incorporate delay times and to improve the regulation or tracking of perturbation trajectories.

In this paper, we propose to use a MPC supervisor, where the MPC controller calculates the optimum references for simple control loops regulated by individual PID controllers, to reduce energy consumption in an industrial process. In order to determine the success of the predictive control strategy, the reduction of the mean square error, the establishment time of the controlled variable, and the process energy consumption resulting of the application of the predictive control topology will be compared against the results obtained when a control strategy exclusively based on PID controllers was used.

\section{PROCESS DESCRIPTION}

The process to be controlled is a scale model of a real pasteurizing plant (Armfield- PCT23 MKII Process Control Trainer) [2]. An schematic of the PCT23-MKII process plant is shown in Fig. 2 and consists of: a miniature 3-stage plate heat exchanger (3) heated from a hot water circulator (4), two independent feed tanks (A or B) (1), a holding tube (2) with product divert valve and two variable-speed peristaltic pumps. Temperature, level, flow and conductivity control loops can be implemented within the plant.

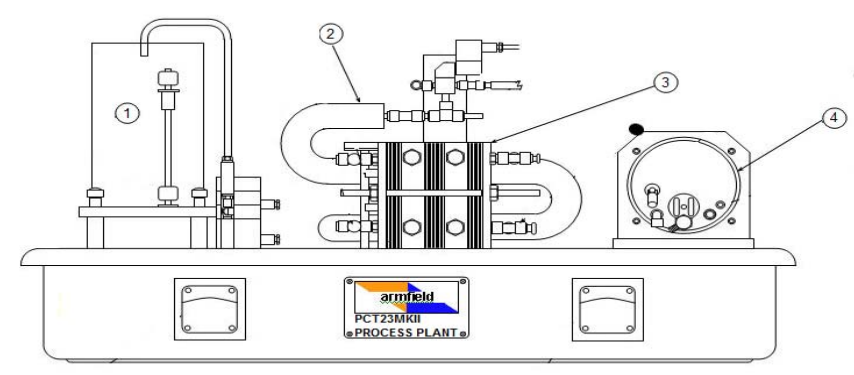

Fig. 1. Schema of the PCT23-MKII process plant [2].

The energy for the pasteurization process comes from the hot water tank, the temperature of which is controlled by the power dissipated in a resistance. This hot liquid is pumped into the heat exchanger to heat the liquid. The process liquid (water) is pumped at a pre-set flow rate from one of the two storage tanks (A or B) to the heat exchanger, where its temperature is raised to a predetermined value. The liquid is maintained at this temperature for a determined period of time using the holding tube, effectively a volume/flow delay or time-out. The fluid exiting the maintenance tube passes through a valve (SOL1) which allows only the correct temperature liquid to advance in the process, the remaining being rejected. 


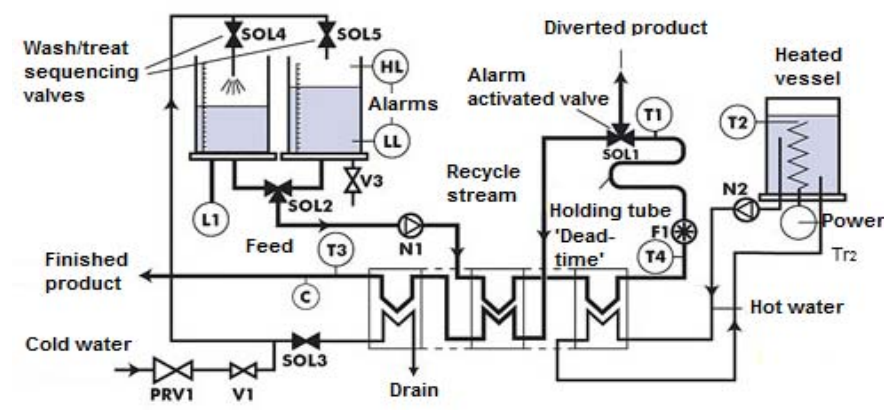

Fig. 2. Process flow diagram for the pasteurizing process [2].

The process fluid advances through the regeneration phase and finally through the cooling phase where it is cooled using cold water supplied from the outside [2]. The process unit has built-in solenoid valves that allow remote control from the control console or from a remote computer, these include: a solenoid valve for deflecting the product (SOL1), a supply solenoid valve selector, either from tank A/B (SOL2), a solenoid valve for cooling the product $(S O L 3)$, a solenoid valve for filling tank A (SOL4), a solenoid valve for filling tank B (SOL5). Two peristaltic pumps are also included: a feed pump (N1) and a hot water supply pump (N2). A pressure reducing valve $(P R V 1)$ and a flow control valve $(V 1)$ control the flow of cold water to the feed tanks and the cooling section of the heat exchanger.

Temperatures are monitored by four temperature sensors (T1, T2, T3 and T4) at key points in the process. The sensor signal of each sensor gives a direct measurement of the temperature in the process pipeline in the range $(0-150)$ ${ }^{\circ} \mathrm{C}$. The usual positions of these sensors provide the following Temperature information: $T_{1}$, located at the product output of the maintenance tube; $T_{2}$ of hot water; $T_{3}$ at the output of the product and $T_{4}$ at the output of the heat product exchanger.

A conductivity sensor $(C 1)$, measures the conductivity of the process fluid in the range $(0-200) \mathrm{mS} / \mathrm{cm}$. This measure will not be used in the process. It would be important to work with saline liquids where the conductivity changes with temperature. A flow turbine type sensor is also installed in the product path to measure the product flow $(F 1)$, at the entrance to the maintenance tube within the range $(0-500) \mathrm{mL} / \mathrm{min}$. An in-line filter is incorporated to prevent debris from entering the sensor. A level sensor $(L 1)$, is connected to a socket at the bottom of tank A. This sensor gives a direct measurement of the level in the tank in the range $(0-250) \mathrm{mm}$. Two float switches LL and HL in tank B are used to detect low and high levels in the tank.

\section{Process Control}

The process addressed in this paper has highly coupled temperature and flow variables. The variation of any of them affects the others. Hence, it is important to set controls for each one of the variables involved. In addition, the presence of disturbances and the imprecision of the models obtained make it necessary to apply controllers in order to achieve the proposed control objectives.

\section{A. Control loops}

The pasteurization plant contains three inputs: Velocity in the pumps $\left(N_{1}\right)$ and $\left(N_{2}\right)$ and the power $(P)$ applied to the resistor of the heather and five measured outputs: $F_{1}, T_{1}, T_{2}$, $T_{3}$ and $T_{4}$. For the temperature $T_{3}$, which is the temperature at which the liquid from the cooling phase exits, there is not a control loop, since the liquid is manipulated by an all-nothing valve, also its control does not have the same importance as the other outputs. For other outputs a control loop is required. The following are the control loops required and the importance of each one of them.

In both cases, the PID and MPC controllers to be implemented, the flow $F_{1}$ must be kept constant for a major reason: in a pasteurization process, the liquid to be treated must be kept in the coil for a certain period of time (ie, pasteurization time). For this time span to be always the same, it is necessary that the flow must be constant. To do this, a PI-type control loop of $F_{1}$ by the speed of $N_{1}$ is needed in order to reject any perturbations and keep the flow constant. One of the disturbances is the wear of the rubber tube of the pump $N_{1}$, another could be caused by the filling process of the tanks A and $\mathrm{B}$ that can introduce air bubbles that affect the flow rate, among others.

On the other hand, the hot water tank is the source of energy for heating the liquid to be treated. The stored energy is expressed in function of its temperature $T_{2}$, therefore it is necessary to control this temperature in order to guarantee the energy supply for the process. The control of $T_{2}$ is achieved by the energy power $P$ applied to the resistance of the heater. The most important variable to control is temperature $T_{1}$. Keeping the product temperature at a value $T_{1 \text { ref }}$ means that the product has been pasteurized correctly. In the opposite case, the product must be subjected to the pasteurization process again and this implies loss of time and additional energy consumption. Temperature control $T 1$ is controlled by a cascade control with an internal control loop of $T 4$, since its value depends strongly on the value of $T_{4}$ (temperature considered at the entrance of the coil). Then, an internal control loop of $T 4$ by $N 2$ is first needed and then an external controller, similar to Fig. 3. In summary, three PI-type control loops are proposed. Two simple control loops and one cascade control loop. The performance indicator used to provide a quantitative measurement of the performance of each controller implemented.

\section{MPC AS SUPERVISORY CONTROL}

The aim of the MPC controller is to improve the performance of the process from an energetic point of view. In this MPC control topology, it is desired to perform a $T_{1}$ trajectory tracking control by means of the PI controls described above, but optimizing the energy consumption, basically in the hot water tank. Fig. 4 shows the configuration used for this topology. The Process Models block contains the models that 


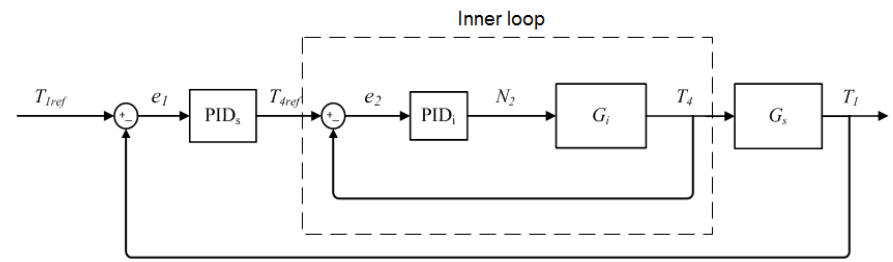

Fig. 3. Scheme of the cascade control for temperature $T_{1}$.

relate the temperatures $T_{2}$ and $T_{1}$ to the set-points $T 2_{\text {ref }}$ and $T 1_{\text {ref }}$, respectively, for the PI control loops. These models are used to calculate the prediction outputs $\left(T_{1}^{*}\right.$ and $\left.T_{2}^{*}\right)$ to $H_{p}$ steps in the future. The predictions are calculated by taking into account the information sampled in the present time $\left(T_{1}(k)\right.$ y $\left.T_{2}(k)\right)$ and also the sequence of applied instructions $\left(T_{1 \text { ref }}\right.$ and $\left.T_{2 \text { ref }}\right)$. The optimization algorithm computes the sequence of $\left\{T_{2 \text { ref }}(k-1+i), i=1, \ldots, H_{c}\right\}$ that minimizes the cost function specified in the problem, subject to constraints. The value of $H_{c}$ is considered equal to $H_{p}$, which gives the maximum possible number of degrees of freedom in the controller. $H_{c}$ and $H_{p}$ are the control and prediction horizons respectively. Following the philosophy of predictive control, only the first element of the optimized control sequence is applied. This is repeated at each sampling instant taking into account the new measurement of the model states (feedback) by recalculating the optimal sequence.

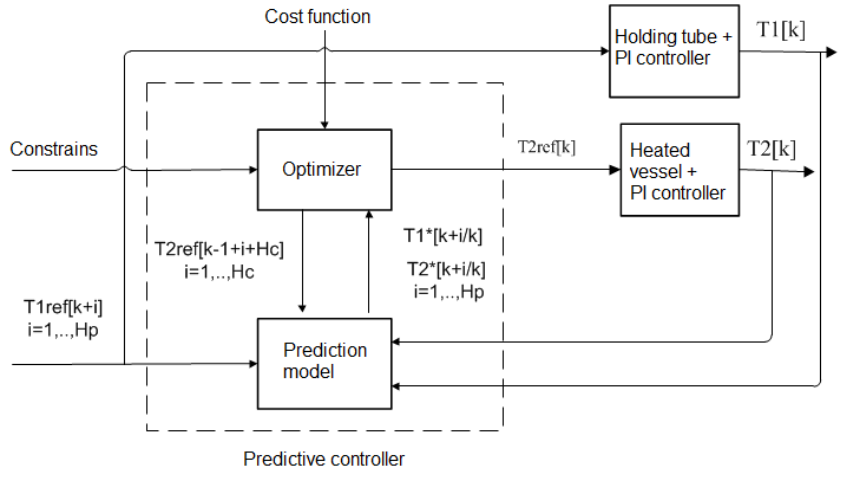

Fig. 4. Model Predictive Control as Supervisor Topology.

The cost function is formed by the control objectives, among which is the minimization of the energy consumption of the process, penalization of the variations of the calculated control actions and the penalization of the term that softens the restriction [3]-[5]. Whereas the restrictions are the conditions that must be fulfilled in order for the PI control of the temperature $T_{1}$ to function properly [6], such as having sufficient energy in the hot water tank.

\section{A. Identification of Models for the MPC Supervisor}

Mathematical models were identified and validated from experimental data from the PI control loops for $T_{1}$ and $T_{2}$ using the Matlab System Identification Toolbox [7], [8]. $T_{2 r e f}$ and $T_{1 \text { ref }}$ set-point were used as input and the temperatures
$T_{2}$ and $T_{1}$ of the PI controls were used as output. Fig. 5 shows the inputs and outputs used for both: model identification and validation of the model that relates $T_{2}$ to $T_{2 r e f} . T_{2 \delta}$ and $T_{2 \delta r e f}$ are the increments of $T_{2}$ and $T_{2 r e f}$ after applying the control action at $\mathrm{t}=10 \mathrm{~s}$. Three models were obtained as candidates for best fit, as it can be seen in the bottom graph of Fig. 5. The models that best approximate are two of the second order $\mathrm{OE}$ type and one delay, and one of the first order ARX type with a delay. Table I, shows the fit values for the Loss Function (Lf) and Akaikes Final Prediction Error (FPE) observed [7]. The model that best approximate is the OE121 model, which is of second order and with a pure delay of 1s. Accordingly, the transfer function that relates $T_{2 \delta}$ to $T_{2 \delta r e f}$ is:

$$
G_{T 2}(z)=\frac{0.00358 z^{-1}}{1-1.937 z^{-1}+0.9406 z^{-2}} .
$$

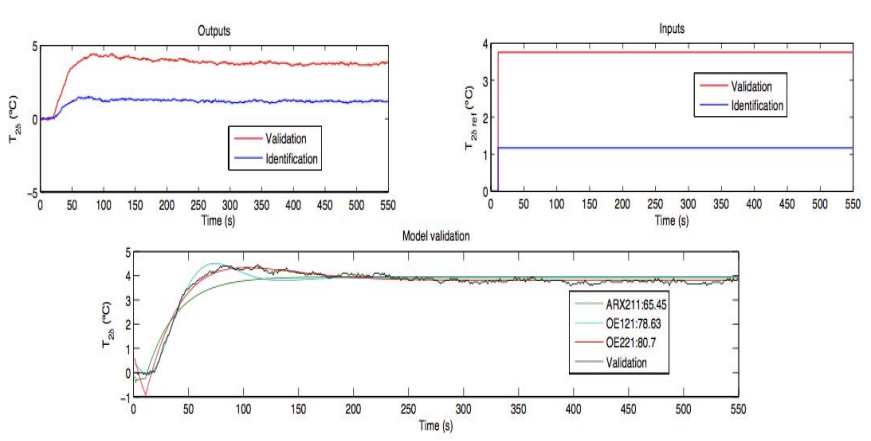

Fig. 5. Upper graphs: data used for the model identification and its validation. Bottom graph: model validation for three structures found.

TABLE I

LF AND FPE VALUES FOR THE MODELS RELATING $T_{2}$ TO $T 2_{\text {ref }}$.

\begin{tabular}{|c|c|c|c|}
\hline & OE121 & OE221 & ARX211 \\
\hline Lf & 0.00526923 & 0.00606671 & 0.00536532 \\
\hline FPE & 0.00532131 & 0.00614641 & 0.00548756 \\
\hline
\end{tabular}

Similarly, Fig. 6 shows the inputs and outputs used for the identification and validation of the the model that relates $T_{1}$ to $T_{1 \text { ref }}, T_{1 \delta}$ and $T_{1 \delta \text { ref }}$ are the increments of $T_{1}$ and $T_{1 \text { ref }}$ after applying the control action at $t=0 \mathrm{~s}$. Two models are best fitted (bottom graph of Fig. 6). The models that are best approximated are of the second order OE type and with a pure delay of $30 \mathrm{~s}$. The setting (Fits) is similar for both models. Table II shows the values observed for the parameters Lf and FPE. Therefore, the OE1230 model was selected and the transfer function that relates $T_{1 \delta}$ to $T_{1 \delta r e f}$ is:

$$
G_{T_{1}}(z)=\frac{0.0008008 z^{-30}}{1-1.963 z^{-1}+0.9639 z^{-2}} .
$$

\section{B. Restrictions for the MPC control formulation}

The energy to heat the product comes from the hot water tank. This energy depends directly on the temperature $T_{2}$. At high temperatures, a large amount of energy will be stored and 

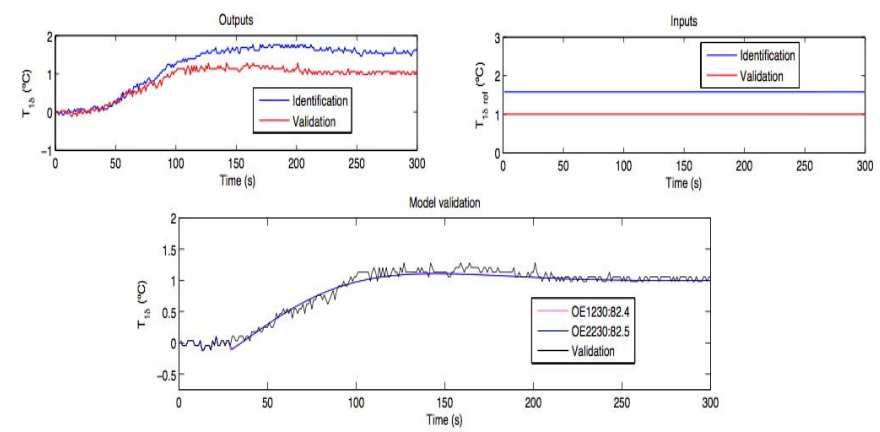

Fig. 6. Upper graphs: data used for the model identification and its validation. Bottom graph: model validation for three structures found.

TABLE II

LF AND FPE VALUES FOR THE MODELS RELATING $T 1$ TO $T 1_{\text {ref }}$.

\begin{tabular}{|c|c|c|}
\hline & OE1230 & OE2230 \\
\hline Lf & 0.00277185 & 0.00277795 \\
\hline FPE & 0.00282708 & 0.00283421 \\
\hline
\end{tabular}

vice versa. Having sufficient energy is fundamental for the PI control of $T_{1}$, hence the MPC Supervisor control algorithm must incorporate this requirement. This requirement is fulfilled by maintaining the temperature $T_{2}$ above the temperature at the output of the serpent $T 1$ at a value $D$, as expressed in the following inequality:

$$
T_{2}(k+i)-T_{1}(k+i) \geq D,
$$

Where $D$ is the minimum temperature difference that must exist between $T_{2}$ and $T_{1}$ so that the control loop PI of $T_{1}$ reaches the desired set-points without having to keep the control inputs in the pump $N 2$ at high values and $i=1, . ., H_{p}$.

For any disturbance in the plant or noise, the difference $T_{2}(k)-T_{1}(k)$ may become less than the value $D$. In that case, the optimizer calculates $T_{2 \text { ref }}$ relatively large with the aim of fulfilling (3), generating therefore oscillations in temperature $T_{2}$. To remedy this effect, a term $\xi$ should be added to make the restriction smooth. Thus (3) can be rewritten as:

$$
T_{2}(k+i)-T_{1}(k+i) \geq D+\xi(k+i) .
$$

For the present design, $D$ will be the temperature difference when the system reaches the working point with $F_{1}=163$ $\mathrm{mL} / \mathrm{min}, N_{2}=65 \%$ and $P=270 \mathrm{~W}$. Therefore, around the working point, we have:

$$
T_{2 \delta}(k+i)-T_{1 \delta}(k+i) \geq \xi(k+i) .
$$

The restriction must be met for each prediction instant, i.e.,

$$
\begin{aligned}
T_{2 \delta}(k+1 \mid k)-T_{1 \delta}(k+1 \mid k) & \geq \xi(k+1 \mid k), \\
T_{2 \delta}(k+2 \mid k)-T_{1 \delta}(k+2 \mid k) & \geq \xi(k+2 \mid k), \\
\vdots & \\
T_{2 \delta}\left(k+H_{p} \mid k\right)-T_{1 \delta}\left(k+H_{p} \mid k\right) & \geq \xi\left(k+H_{p} \mid k\right) .
\end{aligned}
$$

summarizing, in vector form we have:

$$
Z_{T_{2}}(k)-Z_{T_{1}}(k) \geq \Xi .
$$

The vector $Z_{T_{2}}$ contains the outputs $T_{2 \delta}$ through $H_{p}$, i.e.,

$$
Z_{T_{2}}(k)=\Psi x(k)+\Theta U(k),
$$

Where $\Psi=C_{Z} S_{x}$ and $\Theta=C_{Z} S_{u}$. The matrices $S_{x}$ and $S_{u}$ are calculated using the state space found in the modeling of $T_{2 \delta}$ with $T_{2 \delta r e f}$. The matrix $C_{Z}$ is a diagonal matrix whose elements are $C$. The vector $U(k)$ contains the entries $\left\{T_{2 \delta r e f}(k \mid k), \ldots, T_{2 \delta r e f}\left(k+H_{u}-1 \mid k\right)\right\} . x(k)$ is the state of temperature $T_{2 \delta}$ at time $k$.

The vector $Z_{T_{1}}$ is calculated from the transfer function (2) by knowing at the instant $k$ the value of $T_{1 \delta}(k)$ and its setpoints to follow. This function could have been expressed in state space and raise the problem as done for $T_{2}$, however since there is a considerable time delay, the number of states increases, so also the dimension of the matrices for the MPC problem, causing the optimization time to increase considerably.

Replacing the terms described above, we arrive at:

$$
\Psi x(k)+\Theta U(k)-Z_{T_{1}}(k) \geq \Xi .
$$

Defining the vectors:

$\mathcal{U}=\left[\begin{array}{l}U(k) \\ \Xi(k)\end{array}\right], \mathcal{A}=[-\Theta I], \mathcal{B}=\Psi x(k)-Z_{T_{1}}(k)$,

with $I$ been an identity matrix of dimension $H_{p}$, The restriction can be expressed as:

$$
\mathcal{A U} \leq \mathcal{B}
$$

This inequality is one of the constraints of the quadratic optimization problem after the design of the predictive controller.

\section{Cost function}

It is desired that the outlet temperature of the serpent $T_{1}$ follow a reference trajectory, minimizing the power consumption. The energy consuming elements are the $N_{1}$ and $N_{2}$ pumps and the electrical resistance that heats the water in the tank. The speed in the pump $N_{1}$ is directly related to the feeding flow of the product $F_{1}$ and this determines the time the liquid remains in the coil. Because in the pasteurizing process it is necessary for the liquid to be maintained for a certain time in the coil, a constant flow is required, i.e. a constant pump speed $N_{1}$. So the energy optimization will be related to the speed of the pump $N_{2}$ and the power in the resistance, $P$. According to the technical data sheets of the $N_{2}$ peristaltic pump, the energy consumption is approximately $35 \mathrm{VA}$ for flow rates ranging from $100-350 \mathrm{~mL} / \mathrm{min}$ [9], indicating therefore, the low dependence of the electrical power consumed as a function of the flow rate. This leads us to think of optimizing the energy consumption only in the electrical resistance, whose value is about $300 \mathrm{~W}$. The temperature of the hot water tank 
$T_{2}$ is related to the electrical power applied to the resistor. For $T_{2}$ temperatures greater than $65^{\circ} \mathrm{C}$, higher electrical power is required than for $T_{2}$ temperatures below $65^{\circ} \mathrm{C}$, due to factors such as loss of energy to the environment, loss of energy from the rubber in contact with the pump $N_{2}$, etc. Then, minimizing the energy consumption equates to minimizing the temperature $T_{2}$. Therefore, the MPC controller must calculate the minimum $T_{2 \text { ref }}$ set-points for the control loop PI by considering the constraint (9). Thus, the approach to the MPC problem involves the penalization of these parameters. The term that softens the restriction should also be penalized. It is desired that this parameter should be very close to 0 ; how close will depend on the priority it has in the cost function of the multi-objective problem. As such, the variations in the $T_{2 \text { ref }}$ set-points are penalized. This helps to decrease certain oscillations of the temperature $T_{2}$.

Finally, the cost function proposed has the form:

$$
V(k)=\|U(k)\|_{W_{R_{1}}}^{2}+\|\Delta U(k)\|_{W_{R_{2}}}^{2}+\|\Xi(k)\|_{W_{R_{3}}}^{2},
$$

Where $U(k)$ contains the set-points $T_{2 \delta r e f}(k \mid k), \ldots, T_{2 \delta r e f}\left(k+H_{u}-1 \mid k\right), W_{R_{1}}$ is the matrix of weights to penalize $U(k), W_{R_{2}}$ is the matrix of weights to penalize $\Delta U(k)$ and $W_{R_{2}}$ is the matrix of weights to penalize $\Xi$

Considering that:

$$
U(k)=F \Delta U(k)+U_{a},
$$

where: $F$ and $U_{a}$ are defined as:

$$
F=\left[\begin{array}{cccc}
I & 0 & \ldots & 0 \\
I & I & \ldots & 0 \\
\vdots & \vdots & \ddots & \vdots \\
I & I & \ldots & I
\end{array}\right], \quad U_{a}=\left[\begin{array}{c}
u(k-1) \\
u(k-1) \\
\vdots \\
u(k-1)
\end{array}\right],
$$

where $I$ is an identity matrix of dimension $l, F \in$ $\mathbb{R}^{l\left(H_{p} \times H_{p}\right)}, U_{a} \in \mathbb{R}^{l H_{p} \times 1}$.

by defining $F_{1}=F^{-1}, \Delta U(k)$ can be rewritten from (11) as

$$
\Delta U(k)=F_{1} U(k)-F_{1} U_{a} .
$$

therefore, the cost function can be rewritten as:

$$
\begin{aligned}
V(k)= & U(k)^{T}\left(W_{R_{1}}+F_{1}^{T} W_{R_{2}} F_{1}\right) U(k) \\
& -2 U_{a}^{T} F_{1}^{T} W_{R_{2}} F_{1} U(k)+\Xi(k)^{T} W_{R 3} \Xi(k)+\alpha .
\end{aligned}
$$

The term $\alpha$ is a constant, so it does not influence the optimization problem solution. Now, defining:

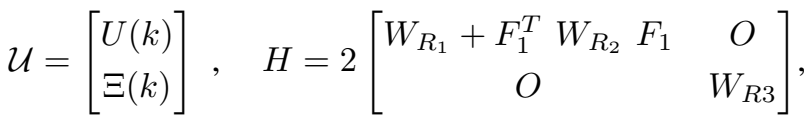

$$
\begin{aligned}
& f^{T}=\left[\begin{array}{lllll}
-2 U_{a}^{T} & F_{1}^{T} & W_{R_{2}} & F_{1} & O
\end{array}\right],
\end{aligned}
$$

we have,

$$
V(k)=\frac{1}{2} \mathcal{U}(k)^{T} H \mathcal{U}(k)+f^{T} \mathcal{U}(k) .
$$

\section{Tuning MPC-Supervisor parameters}

In the MPC it is necessary to tune in certain parameters that will determine the fulfillment of the control objectives. The parameters to tune are basically the weights in the cost function and the control and prediction horizons. For this topology, the control horizon is considered to be equal to the prediction horizon, i.e. $H c=H p$, whereby a greater degree of freedom is obtained in the control actions. The value of the forecast horizon that was applied was $H p=35$. Several horizon values were tested in the simulation. For smaller values the response of the system was slow, whereas for higher values the response in time did not improve remarkably. In the same way, the weights in the cost function were determined by try and error in simulation and then applied to the real system. The tuned values for the diagonal elements of the weight matrices $W_{R_{1}}, W_{R_{2}}, W_{R_{3}}$ are: $R_{1}=0.1, R_{2}=0.1 \mathrm{y}$ $R_{3}=10$. The weight $R_{3}$ at the end of $\Xi$ is about two or more orders of magnitude greater than the others terms because the term that softens the restriction must be close to zero, its only function is to soften the restriction and not to allow very large values of $T_{2 \text { ref }}$ compared to the working temperature (around $65{ }^{\circ} \mathrm{C}$ ), in possible situations where $T_{2}-T_{1}<D$.

\section{RESUlTS AND DiscUSSION}

The optimization problem to be solved for finding the optimum references in $T_{2 r e f}$ is stated by (14) and (9). The experimental results obtained are shown in Fig. 7, where the blue trace represents the temperature value $T_{1}$ that follows the set-point path given by the red curve. As it can be seen, when applying a perturbation at about $550 \mathrm{~s}$ there is a pure delay of about $30 \mathrm{~s}$ which is expected, since no action has been taken to correct it, but the steady-state error tends to zero. At each sampling second, a vector of optimum values $T_{2 \text { ref }}$ is calculated and the first element of said vector is applied to the plant. The $T_{2 \text { ref }}$ values applied to the plant are shown in the orange trace. As it can be seen, as soon as the set-point $T_{1 \text { ref }}$ changes, also $T_{2 \text { ref }}$ changes. The changes are smooth and remain around a certain value, when $T_{1}$ has already reached the desired set-point. Changes in $T_{2 \text { ref }}$ allow temperature $T_{2}$ (in black) to always be above $T_{1}$ at least at a value $D$. This allows to provide the necessary energy so that the PI control loop controlling $T_{1}$ is not affected. The value $D$ is taken as the difference of $T_{2}(k)-T_{1}(k)$ in $t=40 \mathrm{~s}$ (when $T_{1}$ control starts), giving a value $D=11^{\circ} \mathrm{C}$.

In Fig. 8, the results obtained by applying the MPC Supervisor are compared against to the results obtained when applying only the PI control. In the test shown, the temperature $T_{2}$ was maintained at $75^{\circ} \mathrm{C}$ in order to guarantee sufficient energy for trajectory tracking.

As it can be seen from Fig. 8, the control curves of $T_{1}$ with both topologies are practically overlapped and stabilized in the desired set-point, the temperature $T_{2}$ in the MPC topology is kept lower than in the control with PI controllers only. Working with lower $T_{2}$ temperatures represents savings in energy consumption. The percentage of energy saved for the $1800 \mathrm{~s}$ of experimentation was about $10 \%$. In the lower graph, 


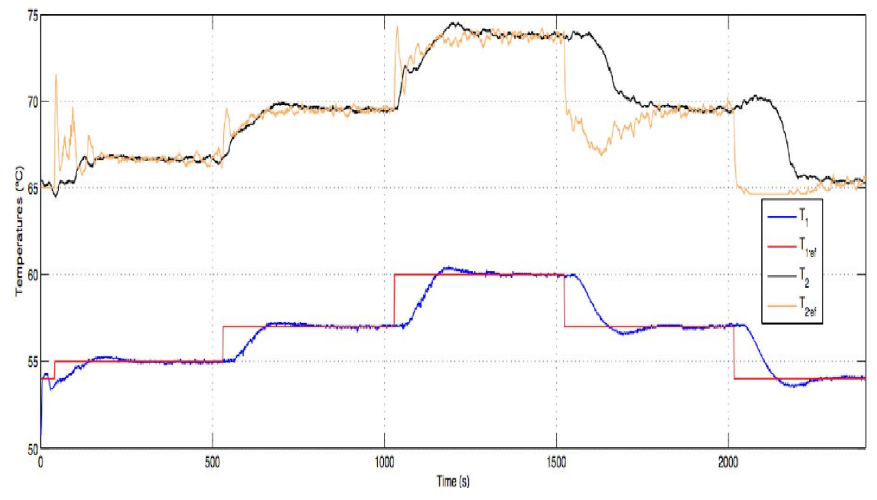

Fig. 7. Evolution of temperatures $T_{1}$ and $T_{2}$ with MPC Supervisory topology.
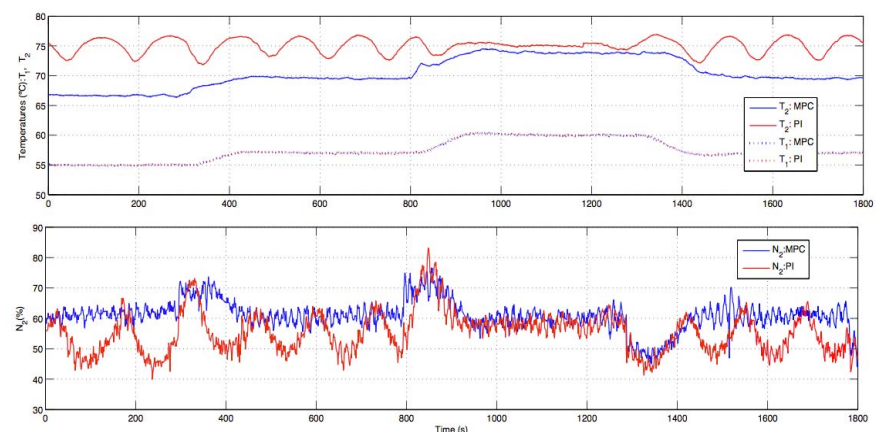

Fig. 8. Upper graph: values of $T_{1}$ and $T_{2}$ for the MPC Supervisor (blue traces) and for control based only on PI controllers (red traces). Bottom graph: speed applied to the pump $N_{2}$ for both control topologies.

the value for the actuator $N_{2}$ is shown for both cases. The red curve has oscillations of considerable amplitude, which affects the $T_{2}$ control. This explains the oscillations of $T_{2}$. Robust controllers should be designed to correct such effect in the future. The mean square error (MSE) obtained for the trajectory tracking of $T_{1}$ when using the supervisor MPC control is 0.94 , which is similar to the value of 0.92 obtained with the PID control.

Table III shows a comparative analysis for the two types of control implemented, based on the steady-state error (mean quadratic error) (MSE), settling time (Ts), and Savings in Energy Consumption (SEC). As it can be seen, the error for the control based exclusively on PID controllers is practically equal to the error obtained by using the supervisor MPC control, even though the latter works with lower temperatures in the hot water tank. This is due to the fact that in the supervisor MPC control, the same PI control loop is responsible for the control of $T_{1}$, as in the case of the PI control based solely on PID controllers. However, by using the MPC control strategy an energy saving of about $10 \%$ was achieved, as it can be seen in Fig. 9.

TABLE III

COMPARISON OF CONTROL STRATEGIES.

\begin{tabular}{|c|c|c|c|}
\hline Topology & MSE & Ts (s) & SEC (\%) \\
\hline based on PID Only & 0.92 & 250 & \\
\hline MPC as Supervisory Control & 0.94 & 250 & 10 \\
\hline
\end{tabular}

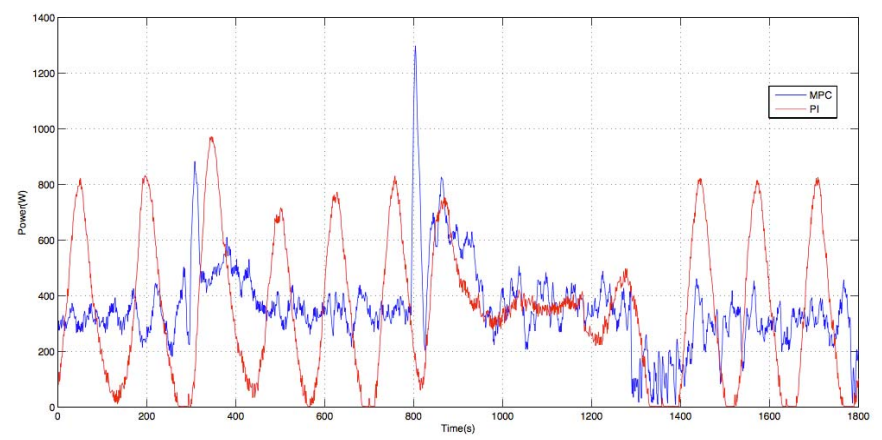

Fig. 9. Heater power consumption for both control topologies. Total energy consumption was computed as the area under the curve.

\section{CONCLUSION}

In this work, it was show that by incorporating energy criteria in the design of control strategies, such as the predictive controller described in this paper, allows the optimal use of resources. The implemented predictive controller allows to reduce energy consumption by about $10 \%$. If these type of controllers are extended to a larger process plant, a similar percentage of energy consumption reduction would imply significant economic savings impact for the industry. The predictive control strategy developed in this paper can be extended to applications of larger plants, since they consider dead zones, delay times and interconnections among subsystems typical of larger scale plants.

We have also investigated the behavior of MPC under other types of configuration topologies, such as MPC multivariable which directly regulates the process variables, and MPC with PID where the PID controls cooperate with the MPC controller, obtaining similar energy saving results, please refer to [10] and [11] for details.

\section{REFERENCES}

[1] E. F. Camacho and C. Bordons, Model predictive control. SpringerVerlag London Limited, 2004.

[2] Armfield, Process Plant trainer PTC23-MKII, Instruction Manual, 2010.

[3] S. Joe Qina and Thomas A. Badgwellb, "A survey of industrial model predictive control technology," Control Engineering Practice, Vol. 11, pp.733764, 2003.

[4] J. Richalet, A. Rault, J. L. Testud, and J. Papon, "Model predictive heuristic control: Applications to industrial processes," Automatica, Vol.14, pp. 413428, 1978

[5] P. Serban, S. Nagy, M. Vacile, and A. Imre. Model Based Control. Wiley, 2006.

[6] J.M. Maciejowski. Predictive control with constraints. Prentice Hall, 2002.

[7] L. Ljung, System Identification: Theory for the User. 2nd. Edition, Prentice Hall, 2007.

[8] L. Ljung. System Identification Toolbox, Users Guide. The MathWorks, Inc, 2016.

[9] Watson-Marlow. Peristaltic Pump Drive Units, 2012.

[10] L. Caiza, "Design and Implementation of Predictive Control Strategies for a Scale Pasteurization Plant - Diseño e Implementación de Estrategias de Control Predictivo para una Planta de Pasteurización a Escala," MS Thesis, Universitat Politécnica de Catalunya, Spain, 2012.

[11] A. Rosich and C. Ocampo-Martinez, "Real-Time Experimental Implementation of Predictive Control Schemes in a Small-Scale Pasteurization Plant," in S. Olaru, A. Grancharova and F. Lobo-Pereira, Eds., Developments in Model-Based Optimization and Control, Distributed Control and Industrial Applications, Lecture Notes in Control and Information Sciences, Vol. 464, pp. 255-273, 2015. 